Research Paper

\title{
Skeletal Muscle-Specific Overexpression of PGC-1a Induces Fiber-Type Conversion through Enhanced Mitochondrial Respiration and Fatty Acid Oxidation in Mice and Pigs
}

\author{
Lin Zhang ${ }^{1}$, Ying Zhou ${ }^{1}$, Wangjun Wu ${ }^{3}$, Liming Hou ${ }^{1}$, Hongxing Chen ${ }^{4}$, Bo Zuo ${ }^{1}$, Yuanzhu Xiong ${ }^{\bowtie}$, \\ Jinzeng Yang1, $2 \bowtie$
}

1. Key Laboratory of Swine Genetics and Breeding of the Ministry of Agriculture, College of Animal Sciences, Huazhong Agricultural University, Wuhan 430070, China;

2. Department of Human Nutrition, Food and Animal Sciences, University of Hawaii at Manoa, Honolulu, Hawaii 96822, USA;

3. College of Animal Science and Technology, Nanjing Agricultural University, Nanjing 210095, China;

4. Beijing Institute of Biotechnology, Beijing 100071, China.

$\square$ Corresponding authors: jinzeng@hawaii.edu, xiongyzy@163.com

(1) Ivyspring International Publisher. This is an open access article distributed under the terms of the Creative Commons Attribution (CC BY-NC) license (https://creativecommons.org/licenses/by-nc/4.0/). See http://ivyspring.com/terms for full terms and conditions.

Received: 2017.03.17; Accepted: 2017.07.17; Published: 2017.09.05

\begin{abstract}
Individual skeletal muscles in the animal body are heterogeneous, as each is comprised of different fiber types. Type I muscle fibers are rich with mitochondria, and have high oxidative metabolisms while type IIB fibers have few mitochondria and high glycolytic metabolic capacity. Peroxisome proliferator-activated receptor gamma coactivator $1 \alpha(P G C-1 \alpha)$, a transcriptional co-activator that regulates mitochondrial biogenesis and respiratory function, is implicated in muscle fiber-type switching. Over-expression of PGC-1 $\alpha$ in transgenic mice increased the proportion of red/oxidative type I fiber. During pig muscle growth, an increased number of type I fibers can give meat more red color. To explore the roles of PGC-1 $\alpha$ in regulation of muscle fiber type conversion, we generated skeletal muscle-specific PGC-1a transgenic mice and pig. Ectopic over-expression of PGC-1a was detected in both fast and slow muscle fibers. The transgenic animals displayed a remarkable amount of red/oxidative muscle fibers in major skeletal muscle tissues. Skeletal muscles from transgenic mice and pigs have increased expression levels of oxidative fiber markers such as $\mathrm{MHCl}, \mathrm{MHC2x}$, myoglobin and Tnnil, and decreased expressions of glycolytic fiber genes (MHC2a, MHC2b, CASQ-1 and Tnni2). The genes responsible for the TCA cycle and oxidative phosphorylation, cytochrome coxidase 2 and 4, and citrate synthase were also increased in the transgenic mice and pigs. These results suggested that transgenic over-expressed PGC-1 a significantly increased muscle mitochondrial biogenesis, resulting in qualitative changes from glycolytic to oxidative energy generation. The transgenic animals also had elevated levels of PDK4 and PPARy proteins in muscle tissue, which can lead to increased glycogen deposition and fatty acid oxidation. Therefore, the results support a significant role of PGC-la in conversion of fast glycolytic fibers to slow and oxidative fiber through enhanced mitochondrial respiration and fatty acid oxidation, and transgenic over-expression of PGC-1 $\alpha$ in skeletal muscle leads to more red meat production in pigs.
\end{abstract}

Key words: PGC-1a; mitochondria biogenesis; fiber type conversion; transgenic pig.

\section{Introduction}

Skeletal muscle is a complex, versatile tissue composed of a large group of heterogeneous fibers, each of which is a multinucleated syncytium developed from myoblast differentiation, and fiber cell fusion. The function of skeletal muscle in the animal body is to support physical movement through transforming the chemical energy derived from ATP hydrolysis into physical force and contraction of the highly organized cytoskeletal apparatus. According to the electrophoretic analysis of myosin heavy chain (MHC) isoforms in adult mammals, skeletal muscle fibers are classified into 
four predominant types: type I with MHC1, type IIA with MHC2a, type IIX with MHC2x, and type IIB with MHC2b [1, 2]. To perform a variety of functional demands during postnatal muscle growth and development, these specialized fiber types have evolved into different bioenergetics and biophysical properties. MHC type I fibers are highly oxidative with the highest content of mitochondria networks, allowing for continuous contractions with less fatigue and favor fatty acid oxidation, a far more efficient ATP production than anaerobic glycolysis [3]. They are almost exclusively found in deep portions of land-dwelling mammalian muscles and tend to be rich in myoglobin and cytochromes, consistent with their role in ambulation [4], for example, the soleus and deep gastrocnemius, their attributes are ideal for the slow, constant role of locomotion [5]. In contrast, type IIB fibers are at the other metabolic extreme as they are poor in mitochondria and depend mainly on glycolysis as the source of energy. Also, they are more susceptible to fatigue compared to oxidative muscles such as the extensor digitorum longus (EDL) and superficial quadriceps, which contain attributes of more amenable to rapid and sudden activity of shorter duration [6,7]. Other fiber types such as IIA and IIX have intermediate biophysical properties and tend to also be oxidative and rich in mitochondria [4]. Skeletal muscle is a dynamic system that has acquired effective adaptive strategies to deal with a myriad of intrinsic and extrinsic stimuli. This remarkable malleability is reflected by the pronounced adjustments in metabolic properties, molecular regulation, endurance and contractile velocity $[8,9$, 10]. Physical activity has particularly profound effects on skeletal muscle physiology. Endurance training induces expansion of the mitochondrial compartment, significant angiogenesis, a fast-to-slow fiber-type conversion which leads to improved endurance performance and resistance to fatigue. Resistance training, on the contrary, induces significant lower mitochondrial amount, a slow-to-fast fiber transformation and a switch to glycolytic energy production system [11]. During fiber-type conversion, the genes coding for MHC isoforms are both temporally and spatially regulated. Recent evidence suggests that MHC transitions follow an obligatory order I $\leftrightarrow$ IIX $\leftrightarrow$ IIA $\leftrightarrow$ IIB [12] .

PGC-1a is originally recognized as a co-activator of PPARY, which plays a profound role in brown fat metabolism during cold exposure [13]. Further studies indicated that PGC-1a is a versatile player, and it interacts with a number of different transcription factors participating in distinct biological programs in various tissues. For example, PGC-1a plays dramatic roles in adaptive thermogenesis in brown adipose tissue [13], angiogenesis in skeletal muscle [14]. It serve as a circadian clock that orchestrates the temporal metabolism in synchrony, and a metabolic feedback signal to regulate tricarboxylic acid cycle (TCA), oxidative phosphorylation, fatty acid oxidation and gluconeogenesis [15, 16]. In addition, an overexpression of PGC-1a in white fat cells leads to a serious attribute of brown fat cells, increased mitochondria and UCP-1 induction [17]. In all of the above cases, PGC-1a displays a dominant role through tight regulation of mitochondrial biogenesis and respiration, leading to a fine-tuning of metabolic homeostasis $[18,19]$.

It has been reported that PGC-1a is implicated in fiber-type switching and determination, causing an increased distribution of red/oxidative fiber types [20]. In pig production, red meat color is preferred by consumers. Meat color is determined by the amount and chemical state of myoglobin. Type I and type IIA fibers have a high content of myoglobin, therefore increasing red color intensity, in contrast, a high proportion of glycolytic fibers results in more white meat production such as in chickens. To understand the scope of fiber-type conversion and metabolic mechanisms from glycolytic to oxdative status of energy production in pig muscle growth and development, our research team initiated PGC-1a transgenic pig project with the intention of increasing red meat production in pigs. This work reported our initial results of the transgenic animals with skeletal muscle-specific over-expressed PGC-1a cDNA sequence. Remarkably, both PGC-1a transgenic mice and swine displayed distinct oxidative properties in major skeletal muscles compared to their littermate wild-type controls, and significant changes in gene expressions associated with fiber-type conversions.

\section{Materials and Methods}

\section{Plasmid construction and transgenic mice}

The vector (MCK-PGC-1a) contains a 7.0-kb porcine MCK promoter, which was obtained by homologous recombination with bacterial artificial chromosome of porcine (Gen Bank accession no. AC139878), and a 2.4-kb complete porcine PGC-1a cDNA (Gene ID: 397013). The Swa1- excised linear vector was microinjected into the male pronuclear of fertilized oocytes from pedigree DBA $\times$ C57BL $/ 6$ hybrid, and MCK- PGC-1a transgenic mice were derived from the microinjected zygotes. Transgenic founders were identified and mated back to C57BL/ 6 wild-type mice to generate F1 offspring animals. Mice were fed ad libitum with free access to food and water. Mice were raised to 4 months of age and tissue 
sampling were conducted for this study.

All mice were maintained in independent ventilation cages (IVC) under conditions of constant temperature $\left(21^{\circ} \mathrm{C}\right)$ and a $12: 12 \mathrm{hr}$ light and dark cycle. All procedures were in compliance with the institutional animal care guidelines and approved by the Institutional Animal Care and Use Committee of Hubei Province.

\section{Southern blot analysis}

Genomic and mitochondrial DNA were isolated from animal tissue (mouse tail tips or pig ear punches) using a DNA kit (Qiagen) according to the manufacturer's protocol, $10 \mu \mathrm{g}$ aliquot of genomic DNA was digested overnight with EcoRI. DNA probe was prepared by PCR and labeled with DIG-dUTP (Roche), the set of primers for probe was forward 5'ACGACGAAGCAGACAAGACCAGTG-3' and reverse 5'- ACGACGAAGCAGACAAGACCAGTG3'. Southern blot was performed according to the manufacturer's protocol of DIG HIGH prime DNA labeling and detection starter kit 1(Roche).

\section{Generation of transgenic pig}

This work was performed by following a previously published protocol of Boquest AC [21]. Briefly, Large White pig fetal fibroblasts were cultured and transfected with the plasmid pMCK-PGC-1a., and stable expression cell lines were established. One male fetal fibroblast cell line was used as donor cells for somatic cell nuclear transfer (SCNT). Large White oocytes collected at a local abattoir were flushed out and matured in vitro, then enucleated the oocytes and reconstructed with the fetal fibroblast transfected with pMCK-PGC-1a. The oocytes were cultured for 3 days after treatment with cytochalasin B and electric fusion/activation. The SCNT blastocysts were transferred to the oviducts of synchronized surrogates. A total of seven surrogates were used for the project, and 39 healthy cloned piglets were delivered after transfer of a total of 238
SCNT embryos. The integration of PGC-1a into chromosomes of the piglets was confirmed by polymerase chain reaction and subsequent qRT-PCR analysis. Transgenic founders (males) were mated back to Large Yorkshire wild-type females to generate F1 spring pigs. Experimental pigs were raised to $90-100 \mathrm{Kg}$ liveweight, and then slaughtered for carcass evaluations and muscle tissue sampling.

\section{Metachromatic dye-ATPase analysis}

Mouse and pig muscle tissues were obtained and oriented for cross sectioning on blocks in OCT compound (Sakara), then frozen in isopentane cooled with liquid nitrogen immediately. Sections were cut in a cryostat $12 \mu \mathrm{m}$ and air-dried prior to the histochemical procedures. All sections were pre-incubated and incubated at room temperature, and $0.1 \%$ toluidine blue $\mathrm{O}$ as a metachromatic dye was used. The different muscle fiber types in a section were distinguished simultaneously according to a method previously described [22].

\section{RNA isolation and quantitative RT-PCR}

Total RNA was extracted from gastrocnemius and quadriceps using a RNA kit (Qiagen) according to the manufacturer's protocol. The first strand cDNA was synthesized from $1 \mu \mathrm{g}$ RNA with RevertAid ${ }^{\mathrm{TM}}$ First Strand cDNA Synthesis Kit (MBI Fermentas, Lithuania). The synthesized cDNA then served as a template for real-time PCR using SYBR PCR Mix (Toyobo, Osaka, Japan) and data were collected in a Bio-Rad C1000TM Thermal Cycler with the CFX96 ${ }^{\mathrm{TM}}$ Real-time PCR System. Real time PCR was performed at $94{ }^{\circ} \mathrm{C}$ for $10 \mathrm{~min}$, and 45 cycles of $94^{\circ} \mathrm{C}$ for $15 \mathrm{~s}$ and $59{ }^{\circ} \mathrm{C}$ for $1 \mathrm{~min}$. GAPDH and beta-actin were used as reference genes to normalize the expression data and calculated with the $\Delta \Delta C t$ method. The primers designed for real-time PCR were listed in Table 1 and Table 2.

Table 1. Primer DNA sequences for Quantitative PCR of mouse RNA samples

\begin{tabular}{lll}
\hline Gene & Forward primer & Reverse primer \\
\hline PGC-1a & CGCAGGTCGAATGAAACTGACTT & GTTACCTGCGCAAGCTTCTCTGA \\
Tnni1 & CTTCAGGACTTGTGCCGAGAG & GCTTGAACTTCCCACGGAG \\
Tnni2 & GAAGATCGACGTGGCTGAAGAG & ACTTGCCCCTCAGGTCAAATAG \\
COX2 & ATCCCAGGCCGACTAAATCAAG & AGAGCATTGGCCATAGAATAAC \\
PDK4 & AGGATTACTGACCGCCTCTTTAG & ATTCCGGGAATTGTCCATCACAG \\
CS & AACTCAGGACGGGTTGTTCCAG & TAGTAATTCATCTCCGTCATGCC \\
Mb & CTGTTTAAGACTCACCCTGAGAC & CTGCCGAGATCCAGCCTCTA \\
COX4 & CTATGTGTATGGCCCCATCC & CAGCGGGCTCTCACTTCTTC \\
CASQ1 & AGAGCCTATGACCATCCCAGAC \\
MYH2X & CCAATGAAACCAAGACTCCTGG & TGTGGATTCCATCCAGGTCATC \\
MYH2B & ACAGACTAAAGTGAAAGCCTACAA & TGCTATCGATGAACTGTCCCTC \\
MYH1 & CAATGGCAAGACGGTGACTGTG & CACATTTTGTGATTTCTCCTGTCAC \\
MYH2A & ATCAACCAGC AGCTGGACAC CA & GGA AGC GTA GCG CTC CTT GAG \\
GAPDH & AAGGTCGGTGTGAACGGATTTG & TCCAGCACGAACATGTGGTGGT \\
\hline
\end{tabular}


Table 2. Primer DNA sequences for Quantitative PCR of pig RNA samples

\begin{tabular}{lll}
\hline Gene & Forward primer & Reverse primer \\
\hline Actin & GTGTTGAAGGTCTCGAACATGAT & CTGGCACCACACCTTCTACAA \\
PGC-1a & CGCAAGCTTCTCTGAGCTTCTTT & GGATACACTTTGCGCAGGTCGAA \\
CASQ-1 & GTGTGGTCAACGTCAACGCAAA & GAAGCCAACACCCTTGTCTTCTA \\
COX5B & CTATGGCATCTGGAGGTGGTGT & CTATCCGCTTGTTGGTGATGGA \\
COX6A & GCCGAAGTGATCTAGGTCGAAA & CCACGAAATAGGTGAGGGCTT \\
COX6B & GTCACATTGAGCTTCCAGCGGT & AGCAGTCATTGCTTTCTCACAGC \\
COX6C & GCCAAGCGTCTGCGATTTCATA & GCACTCTGAAAGATACCAGCCT \\
CS & GGAAGTGCTTGTTTGGCTGACA & CATGAGGCAGGTGTTTCAGAGCA \\
Mb & AGCACCTGAAGTCAGAGGATGA & TCCAGGTACTTGACAGGGATCT \\
MYH1 & GAGGAAGCGGAGGAACAATCCA & GACCTGGGACTCAGCAATGTCA \\
MYH2A & GATGGAGATCGACGACCTTGCT & CTGCTGCTCTTCCTCCTTGGAT \\
MYH2B & CGCCAAGCTACTGAGGCAATAA & GTTCCACCATGGCCAGTTGTTC \\
MYH2X & GAGGAAGAGTGAGCGTCGCAT & ACCTTCAGCTGTAGCTTGTCCA \\
PDK1 & CATATCACGCCTTTATGCACAGT & CAGCCTCATGGTTGGTGTTGTA \\
PDK4 & GCCAGGATATGGAACGGATGCT & GCTTGGGATACACCAGTCACCA \\
Tnni1 & GCTCTAAACACAAGGTGTCCAT & GCCTCGACGTTCTTTCTCCAGT \\
Tnni2 & GGAGAAGCAGAACTACCTGTCT & GGACCTTGATCTCCATGTCGTA \\
\hline
\end{tabular}

\section{Western blot analysis}

Total protein isolations from EDL, gastrocnemius, and quadriceps were prepared by standard procedures and assessed by microplate protein assay (BSA). 100 micrograms of protein per sample were separated on $12 \%$ separation gel and $5 \%$ concentrated gel were used in sodium dodecyl sulfate/polyacrylamide gradient gel electrophoresis followed by transfer onto an Immun-Blot ${ }^{\mathrm{TM}}$ polyvinylidene fluoride (PVDF) membranes (Millipore, Bedford, MA) using BIO-RAD Mini protein tetra system. All antibodies used were commercial antibodies, including HRP-IgG (Abcam, ab6721), anti-PGC-1a (Abcam, ab54481), anti-PPAR- $\gamma$ (Abcam, ab45036), anti-Tubulin (Abcam, ab6046), anti-Tnni2 (Abcam, ab97711), and anti- SERCA1 ATPase (Abcam, ab92666). Immunoreactivity was detected with Immobilon Western Chemiluminescent HRP-DAB substrate (Tiangen, Beijing, China).

\section{Statistical analysis}

The data for mean comparisons were analyzed by subjecting to general linear model of SAS (SAS Inst., Cary, NC). Significant differences of gene expression levels between genotypes (transgenic vs wild-type) were determined by two-tailed student's $\mathrm{t}$-test. All values are expressed as the means \pm SEM of three to six samples per group and have at least three independent trials. Gene expression levels from qPCR data were considered significant difference between genotypes if the mean absolute fold change was larger than 1.0 and the p-value was less or equal to 0.05 .

\section{Results}

\section{Generation of PGC-1 $\alpha$ transgenic mice and their morphological analysis}

The transgenic animal project was designed to promote tissue-specific expression of PGC-1a in skeletal muscle under the control of the MCK promoter, which has been used for tissue-specific gene expression in skeletal muscle [23]. The transgenic mice exhibited the distinct characteristics of oxidative fiber type with strong red color intensity. The effects of transgenic expression of PGC-1a are noticeable in gastrocnemius and soleus muscles in a gross morphological perspective when compared with their wild-type littermates (Fig. 1A). It is also interesting to note that the transgenic mice appear to have less abdominal adipose tissue. The differences are most pronounced in gastrocnemius muscle, which is predominantly composed of fast-twitch type IIB fibers (Fig. 1B and C) in wild-type pigs. The extrinsic appearance of the gastrocnemius muscle in transgenic mice was so red that it showed similar coloration as the red/oxidative soleus muscle. In wild-type littermate animals, soleus muscle is obviously distinguishable from the other muscles due to its red color. As shown in Fig. 1D, the results from DNA Southern blot identified the insertion of transgene to the genome. Founder animals showed a different copy number of the transgene. The offspring pigs from founder line 24 and 9 appear to have a high copy number of the transgene.

\section{Increased type I fibers in gastrocnemius by metachromatic dye-ATPase staining}

Metachromatic staining can simultaneously identify the discrepancy between MHC1, MHC2a and $\mathrm{MHC} 2 \mathrm{~b}$ fibers [22]. To investigate whether there are fiber-type differences in gastrocnemius muscles of the PGC-1a transgenic mice, we evaluated the ratio of slow versus fast fibers by using metachromatic dye-ATPase staining method. As shown in Fig. 1E, there were obvious morphological differences between the wild-type and transgenic mice in fiber 
type composition. The percentage of type I fibers in gastrocnemius muscle was significantly increased from $5 \%$ to $50 \%$ of the total fibers in the transgenic mice compared to the wild-type littermate controls. In the meantime, MHC type IIA fiber was reduced from $50 \%$ to $8 \%$ of total fibers in the gastrocnemius muscle of transgenic mice (Fig. 1E).

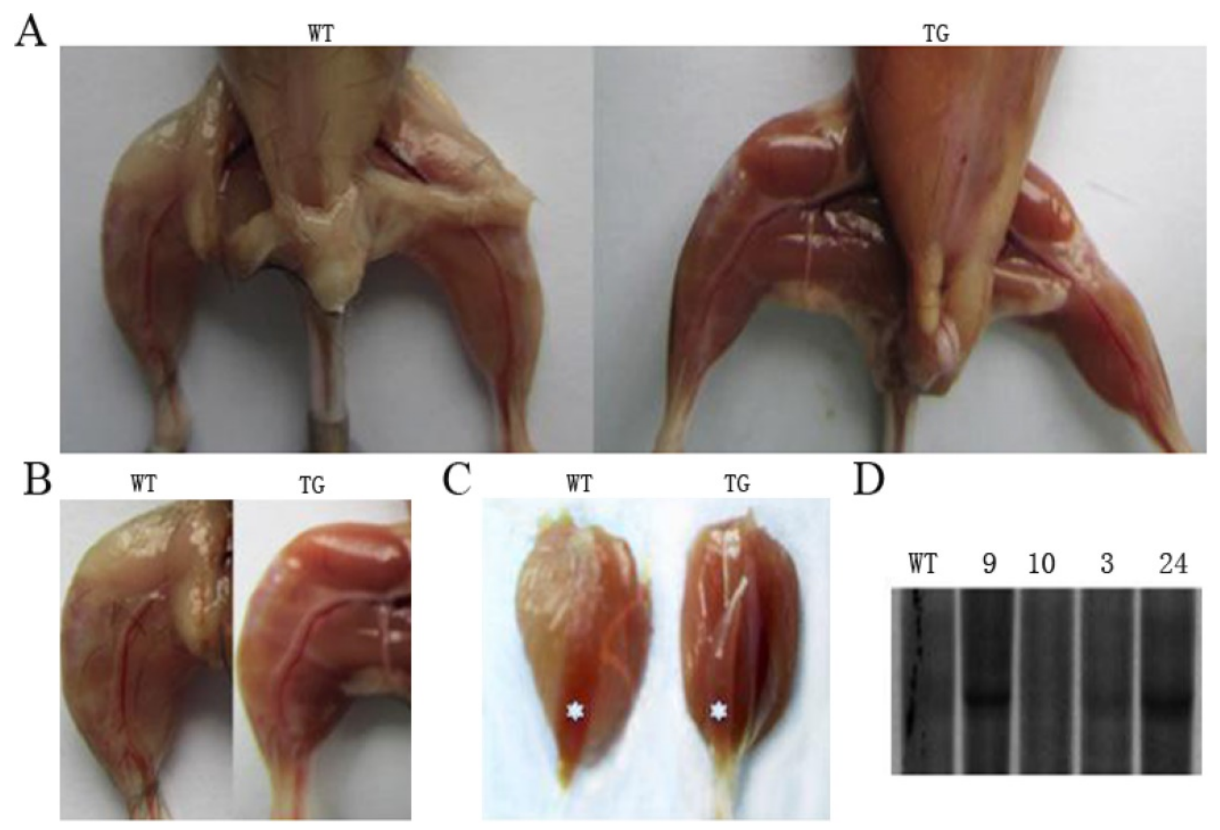

E

WT

TG
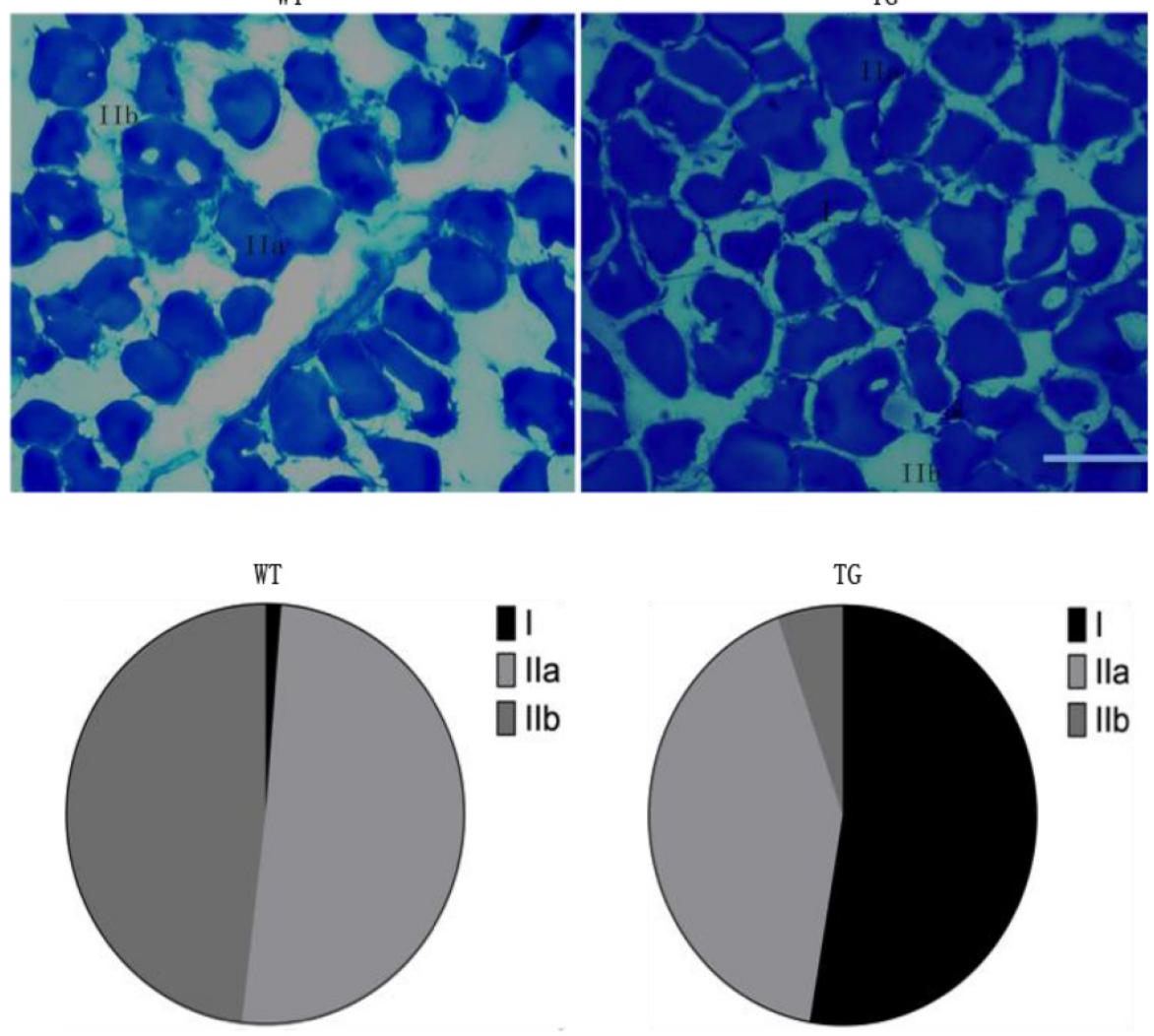

Figure 1. MCK-PGC-1 $\alpha$ fusion gene is over-expressed in skeletal muscles of the transgenic mouse. Type-1 fibers (red) are increased in whole body (A), hindlimb (C) and especially in gastrocnemius/soleus (C with asterisk) muscles by transgenic overexpression PGC-la. Mice were sampled at the age of four months from the founder/line 24 and a wild type littermate. The copy number of MCK-PGC-1 $\alpha$ transgene was analyzed in transgenic founder/Line 3, 9, 10 and 24 and wild-type mice by Southern blot (D). Metachromatic ATPase staining and fiber type analysis of frozen sections from WT and TG gastrocnemius muscle were shown in E, bars is indicated as $50 \mu \mathrm{m}$. After staining with toluidine blue $\mathrm{O}$ as a metachromatic dye, the dark blue color is typically type I fiber, light blue color is stained as homogeneous type IIA fiber, and unstaining with least density mostly are type IIB fiber. Fractions of different fiber types of gastrocnemius muscle, as determined by counting fibers in WT and TG mice. 


\section{Enhanced expressions of the genes for mitochondrial oxidative metabolism and fatty acid oxidation in the PGC-1 a transgenic mice}

To further evaluate the metabolic mechanisms of increased red/oxidation fiber formation and growth in the transgenic mice, we analyzed the expression levels of PGC-1a and several genes involved in oxidative metabolism in skeletal muscle by qPCR method. As shown in Fig. 2A and B, PGC-1a mRNA was significantly elevated in gastrocnemius and quadriceps muscles by more than 18 folds compared with littermate controls. A remarkably increase of cytochrome c oxidase 2 (COX2) and COX4, which are mitochondrial enzymes involved in electron transport, was observed in gastrocnemius and quadriceps in comparison with wild-type littermates. Citrate synthase (CS), the initial enzyme of the tricarboxylic acid cycle (TCA) and an exclusive marker of the mitochondrial matrix [24], was also increased in both gastrocnemius and quadriceps muscles (Fig. 2A and B). In addition, PDK4, a gate-keeping enzyme that phosphorylates and inactivates pyruvate dehydrogenase in mitochondria, has the same trend of expression levels as CS in gastrocnemius and quadriceps muscles.
A

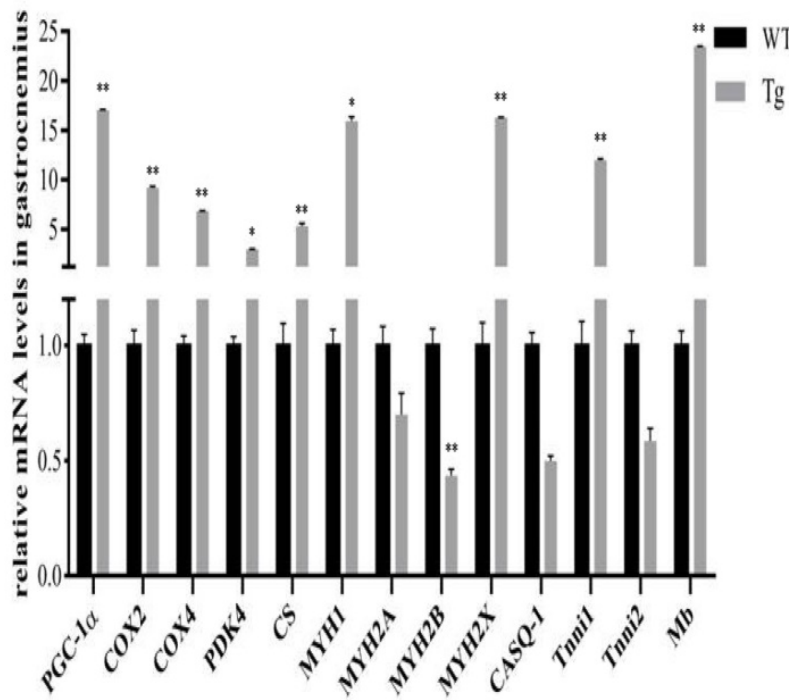

C

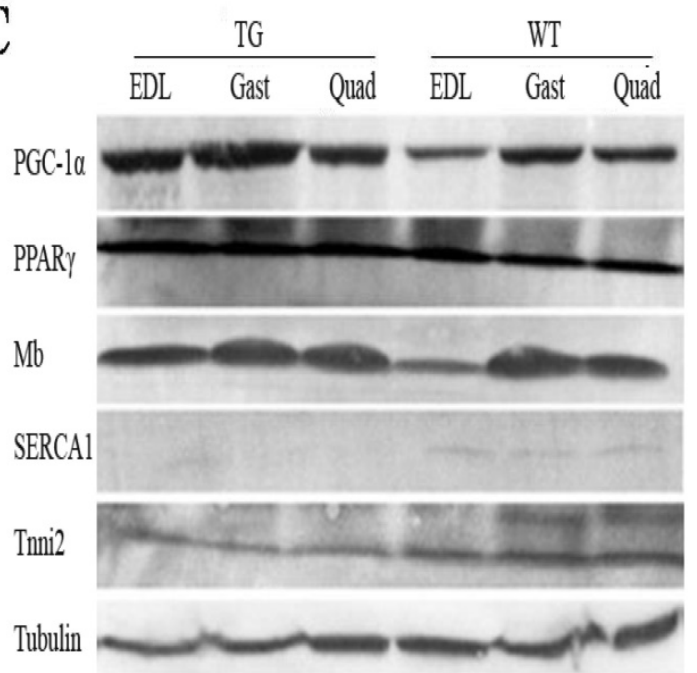

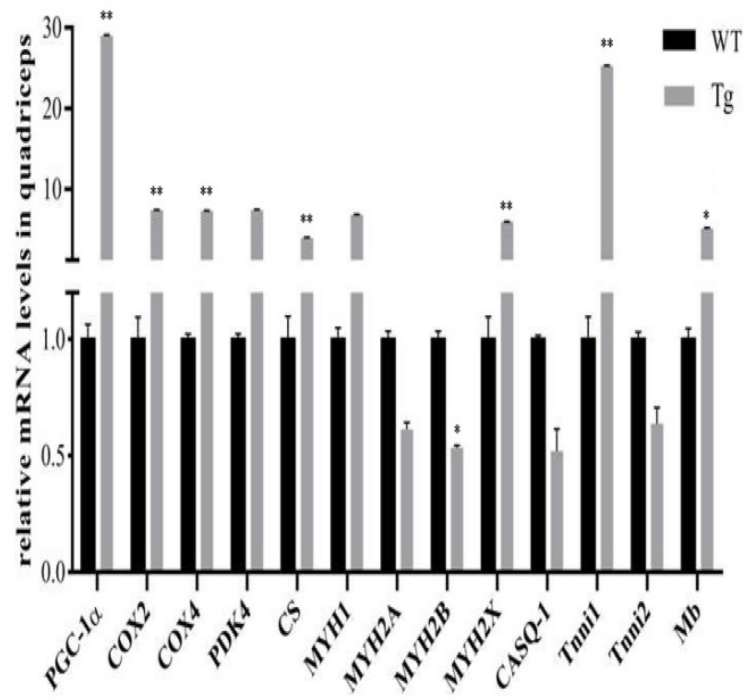

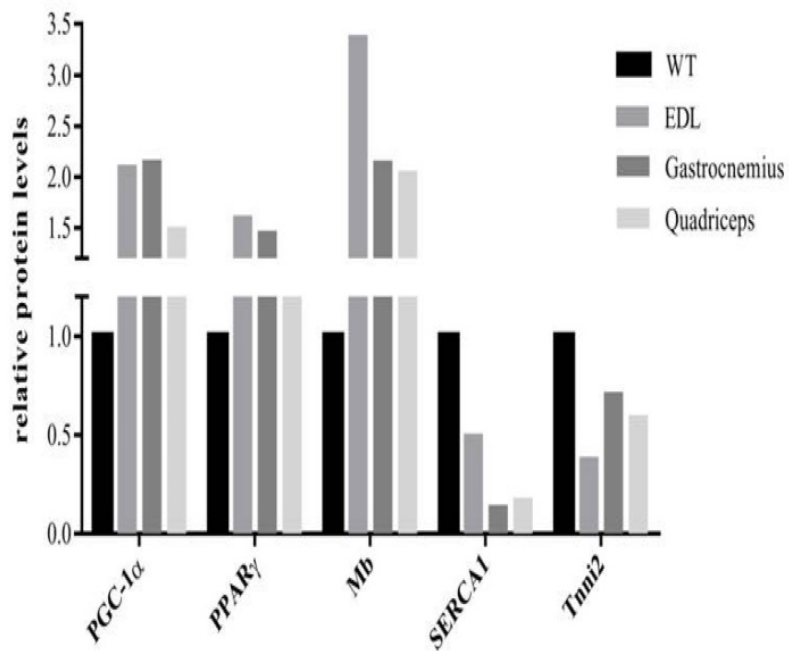

Figure 2. Effects of transgenic overexpression of PGC-1 of the genes responsible for mitochondrial function and fiber type in mice. Relative mRNA expression levels in gastrocnemius (A) and quadriceps (B) muscles of the four-month-old mice of the transgenic mice versus wild-type littermates. Data are presented as means \pm SEM; $\mathrm{n}=3 ; *_{\mathrm{p}}<0.05 * * \mathrm{p}<0.01$. Western blot analysis of oxidative and glycolytic fiber marker genes in type-Il-rich muscles: EDL, gastrocnemius and quadriceps muscles with tubulin as reference for equal loading $(C)$. Analysis of intensities of the proteins bands are corrected by intensities of the tubulin and bar graphic is presented on the right. 
Studies have reported classification of muscle fiber types on the basis of MHC isoforms, and MYH proteins were correlated consistently with mRNA transcript levels [25]. As shown in Fig. 2A and B, the expression levels of a number of oxidative fiber markers, including $\mathrm{MHC} 1, \mathrm{MHC} 2 \mathrm{x}$, myoglobin and Tnni1, were strongly induced by PGC-1a transgene, and a corresponding decrease in the expression level of glycolytic fiber marker genes-MHC2a, MHC2b, CASQ-1 and Tnni2 in quadriceps.

To further confirm the functional role of PGC-1a in transformation of skeletal muscle fiber types, Western blot was used to detect the changes of protein levels in EDL, gastrocnemius and quadriceps. As shown in Fig.2C, the expressions of PGC-1a was strongly elevated in all three MYH type II muscles in transgenic mice, which is 2.3 folds of the littermate control animals. $\mathrm{Mb}$ was also significantly affected by PGC-1a with a 2.6-fold changes compared to the wild-type littermates, which was consistent with the qPCR results. Interestingly, we found that expression of PPARY is also higher in the transgenic MYH type II fibers than that in the wild-type mice, which can lead to an increased uncouple capability of lipid in skeletal muscle. Whereas, the protein levels of other muscle regulatory factors such as Tnni2 and SERCA1were repressed. In particular, SERCA1, usually enriched in MHC type II fibers, were reduced to an undetectable level in the transgenic mice.

\section{Increased type I fiber in gastrocnemius of PGC-1 $\alpha$ transgenic pigs}

The PGC-1a transgenic mouse model provided a solid evidence for an transgenic pig project. The PGC-1a transgenic pigs were generated by SCNT method. In line with morphological characteristics of the transgenic mice, the glycolytic fiber-type musclelongissimus dorsi and gastrocnemius showed a deeper red property when compared with their wild-type littermates. The red color in these muscles were easily distinguished from the wild-type control animal (Fig.3A). Intramuscular fat content, serving as one vital parameter of meat quality that has positive effects on taste and flavor, and is routinely evaluated by "marbling" structure, was surprisingly low in the muscle of the transgenic pigs (Fig.3A). We observed the same tendency in gastrocnemius muscles of the PGC-1a transgenic pigs. As displayed in Fig.3B, the percentage of $\mathrm{MYH}$ type I fibers of gastrocnemius muscle was increased from $5 \%$ to $65 \%$ of the total fibers in the transgenic pigs compared to their wild-type controls. In contrast, MYH type IIA fiber was reduced from $65 \%$ to $25 \%$, and MYH type IIB fiber was reduced from $30 \%$ to $7 \%$ of total fibers. The results of the skeletal muscle phenotype in the transgenic pigs are consistent with PGC-1a transgenic mice.

\section{Gene expressions in PGC-1 $\alpha$ transgenic pigs}

To further evaluate the metabolic mechanisms of increased red/oxidation fiber formation and growth in the transgenic pigs, PGC-1a and oxidative metabolism-related genes were detected by qPCR. As shown in Fig. 4A and B, PGC-1a mRNA level was significantly elevated in gastrocnemius and quadriceps muscles of transgenic pigs. Skeletal muscle COX6b, MYH2X and Tnni1showed similar expression patterns of increased levels in PGC-1a transgenic pigs. On the contrary, MYH2B's expression was distinctly lower in the transgenic pigs than that in the wild-type controls. Similar expression trends were noticed by protein level in the Western blotting analysis. As shown in Fig. 4C, the expressions of PGC-1a was also dramatic elevated in gastrocnemius muscle of transgenic pigs with 2 folds higher than that in the littermates controls. Mb, MHC1, MHC2X, MYH2A were also induced to high levels in the transgenic pigs individually compared to the wild-type littermate controls.

\section{Discussion}

PGC-1a plays a pivotal role in the complicated phenotypic switch of muscle fiber type. It is thought to regulate a core program of mitochondrial biogenesis and an ancillary program of myofibrillar content [4]. This study demonstrated that skeletal muscle-specific expression of PGC-1a transgene in mice and pigs displayed a remarkable amount of red/oxidative muscle fibers in major white skeletal muscle tissues. Transgenic over-expression of PGC-1a increased the expression levels of oxidative molecular markers, such as MHC1, MHC2x, myoglobin, and troponin1, and concurrently significant attenuations of glycolytic fiber marker genes, including MHC2a, MHC2b, CASQ-1, SERCA1 and troponin2 were evident. These results suggest that over-expressed PGC-1a by the transgene construct causes conversions of MHC Type IIB fibers to Type I fibers in several significant skeletal muscles. These results are consistent in line with a study that reported PGC-1a increased the proportion of fibers expressing MHC1and MHC2a, and induced type I fiber genes-troponin I slow and myoglobin [26]. Transgenic overexpression of PGC-1a not only induced the transcriptional activity of a series of genes related to TCA and oxidative phosphorylation such as Cox2, Cox4 and CS, but also generated obvious differences in morphological changes of hindlimb and gastrocnemius and soleus muscles (Fig.1 and 3). We believe that mitochondrial biogenesis was 
dramatically enhanced through oxidative metabolisms in the skeletal muscle of the PGC-1a transgenic animals. It is in agreement with the mitochondrial biogenesis triggered by pharmacological activation of the PPAR pathway through administration of bezafibrate [27]. Isolated mitochondria from muscle with ectopic overexpression PGC-1a also increased respiration caused by complex III and I [28]. There is growing consensus that PGC-1a modulates not only mitochondrial fusion and fission, but also mitochondrial adaptation to changing physiological conditions $[29,30]$. Taken together, these findings indicate that PGC-1a plays a key role in the regulation of aerobic metabolism and homeostasis of MHC isoforms in skeletal muscle. Over-expressed PGC-1a in the transgenic animals can drive glycolytic fast-twitch fibers conversion into more oxidative slow-twitch fibers, which is expected to provide beneficial effects on constant locomotion and endurance training.
A
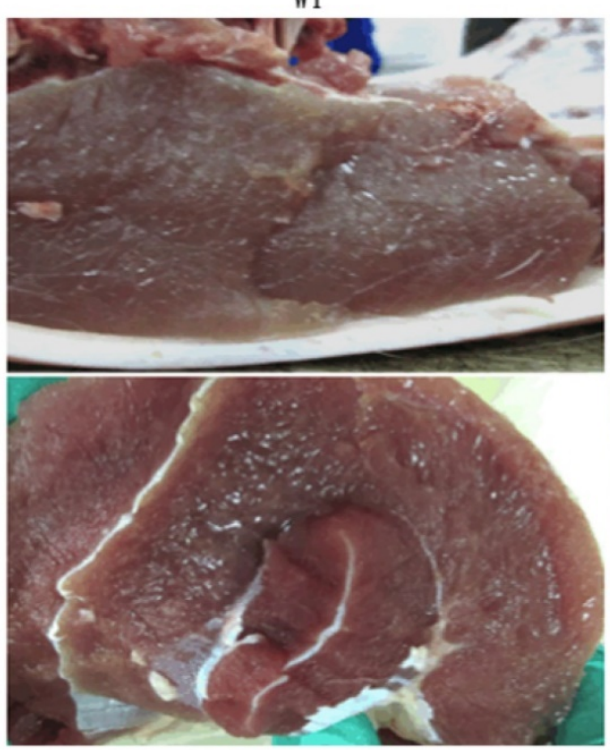

B

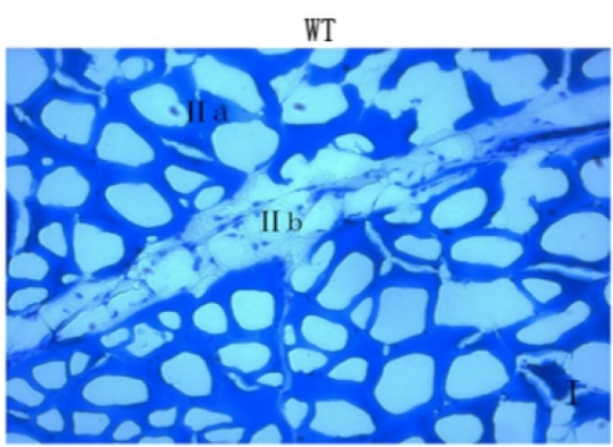

WT

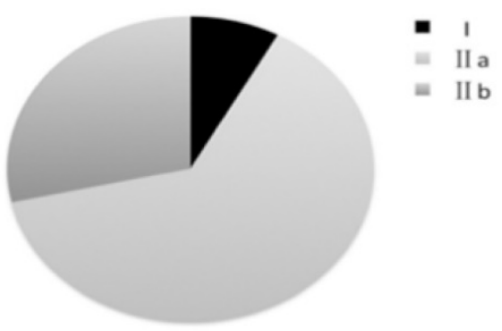

TG
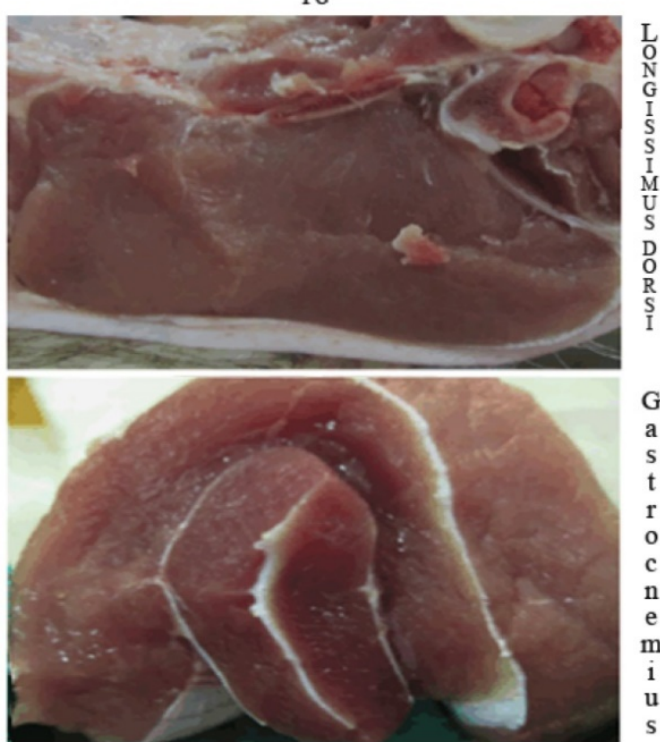

TG

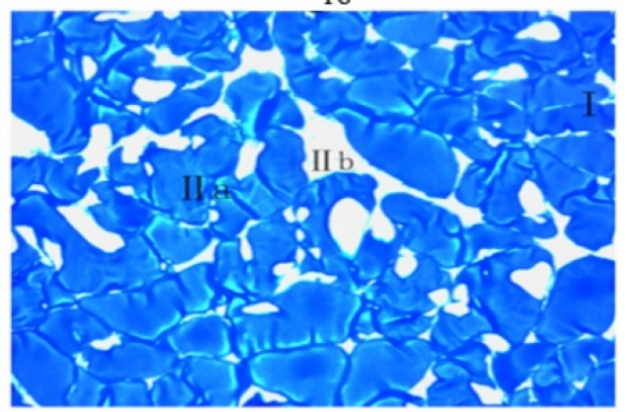

TG

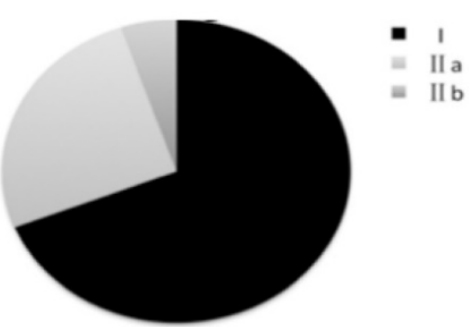

Figure 3. Morphological analysis of skeletal muscle in the MCK-PGC-1 atransgenic and wild-type control pigs. Morphological analysis of longissimus dorsi muscle and gastrocnemius muscle by transgenic expression PGC-1 $\alpha$ (A). The weight of pig is between $90 \mathrm{~kg}$ to $100 \mathrm{~kg}$. Metachromatic ATPase staining and fiber type analysis of frozen sections of gastrocnemius muscle from WT and TG pigs. (B). Fractions of different fiber types of gastrocnemius muscle, as determined by counting fibers in WT and TG pig. 
A

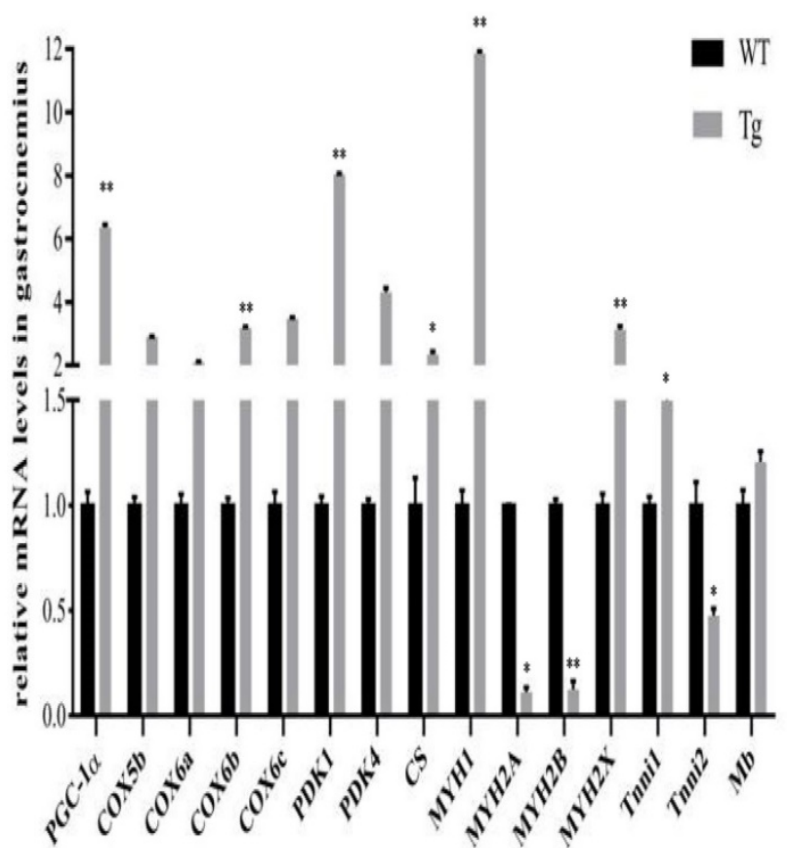

C

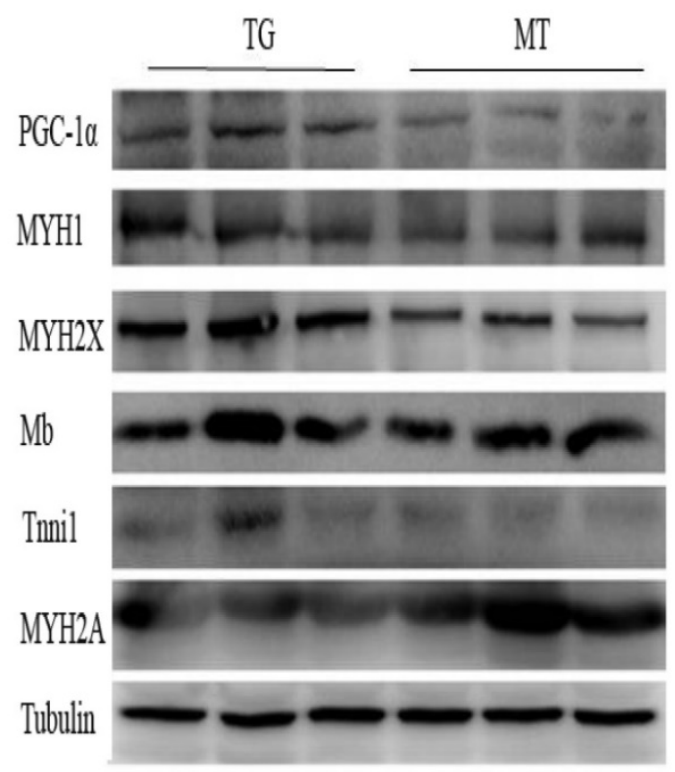

B

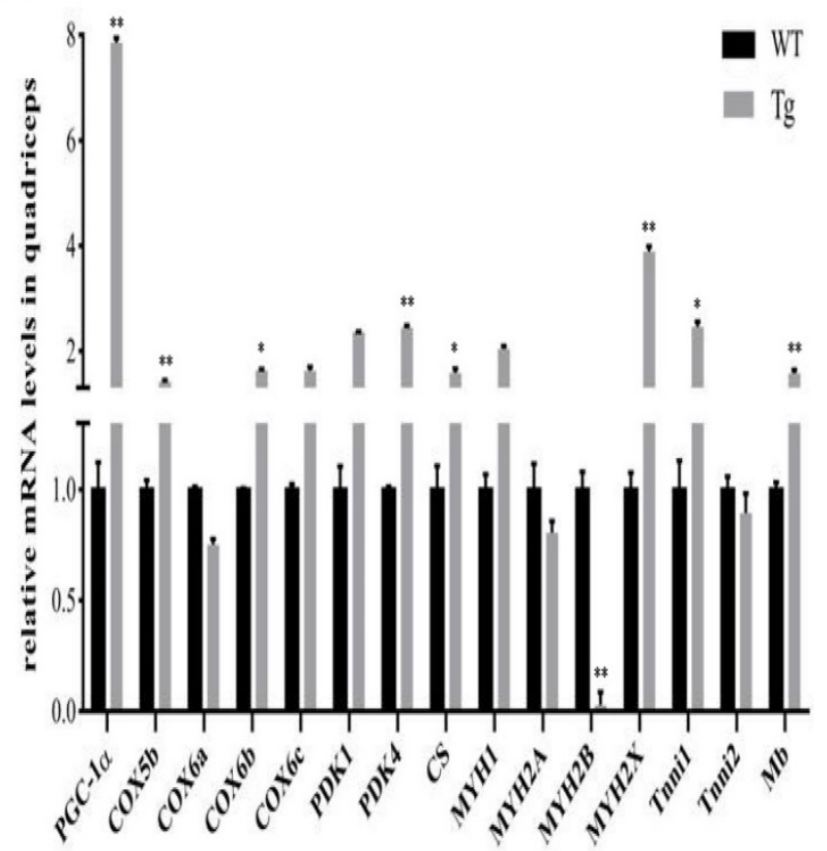

Figure 4. Expressions of PGC-1 $\alpha$ and related genes by qPCR in transgenic pigs. Relative mRNA expression levels were analyzed by qPCR for gastrocnemius (A)and quadriceps muscles (B) from pig at body weight of 90 to $100 \mathrm{~kg}$. Data are presented as means \pm SEM of three to six samples per group and are expressed relative to the mean value of the control, one-way ANOVA significance. ${ }^{*} \mathrm{p}<0.05 * * \mathrm{p}<0.01$. Western blot analysis of oxidative and glycolytic fiber marker genes (C) in type-II-rich gastrocnemius muscles with tubulin as reference for equal loading. Analysis of intensities of the proteins bands are corrected by intensities of the tubulin.

Skeletal muscle is a major player of glucose homeostasis in normal metabolic status and physiologic adaption to physical activity. Cellular glucose utilization is tightly regulated at multiple levels, including uptake by glucose transporters and entry of pyruvate into the citric acid cycle. We observed that transgenic expression of PGC-1a is significantly correlated to the PDK-4 expression. This

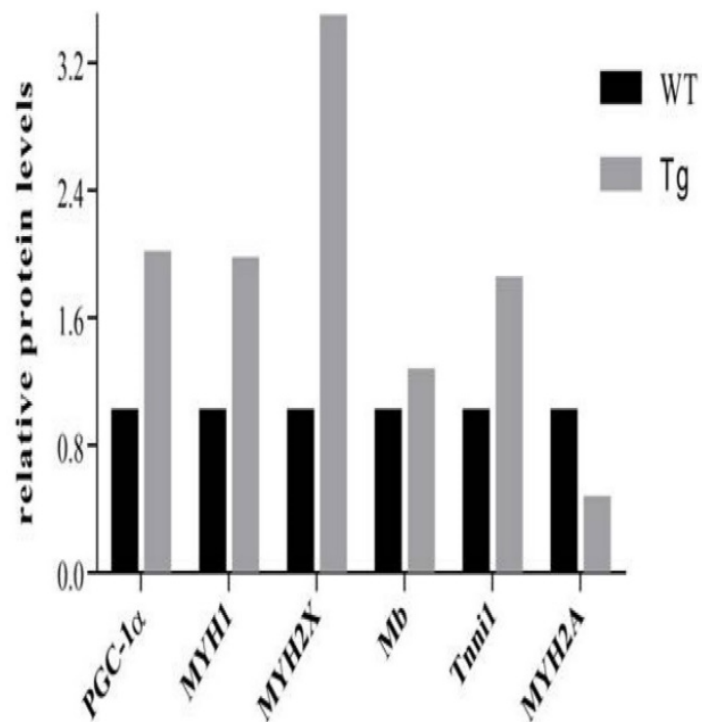

result is consistent with previous report that PGC-1a co-activated PDK4 gene expression via the orphan nuclear receptor ERR alpha [31]. It is demonstrated that PDK-4 phosphorylates and inactivates pyruvate dehydrogenase (PDH) enzyme, which serves as a critical, rate-limiting step in the glucose oxidation pathway by catalyzing the irreversible decarboxylation of pyruvate to acetyl coenzyme A 
[32]. It is interesting to notice that PGC-1a has also been reported to induce expression of the dominant glucose transporter GLUT4 and glucose-6phosphatase in skeletal muscle, both of them are critical genes involved in gluconeogenesis $[33,34]$.

In addition, the expression of a set of genes for aerobic metabolism are quantitatively increased in skeletal muscles of the transgenic animals (Fig. 2 and 4). As a result, this would be accompanied by a significant elevation of bioenergetics consumption. The enhanced respiration requires fatty acids as dominant substrates (palmitoyl co-A with carnitine) during glucose oxidation is blocked by PDK4 [32], and pioneering studies demonstrated that glucose oxidation is decreased coincidently with increased fatty acid oxidation in heart muscle [35]. Together with the rise of PPAR $\gamma$, a critical regulator of insulin sensitization and thermogenesis in BAT [36, 37], it is tempting to speculate that elevated activity of PGC-1a in skeletal muscle might provide beneficial mechanism of crosstalk between glucose metabolism and fatty acid oxidation. We believe that PGC-1a may serve to replenish glycogen levels in muscle by sparing glucose and favoring fatty acid oxidation, a shift in fuel usage from glucose to fatty acid by peripheral tissues. This can provide a critical mechanism for the maintenance of systemic glucose homeostasis and protect against excess accumulation of adipose tissue. The ability of the PGC- 1 a to orchestrate energy homeostasis presented here thus support the idea that PGC-1a is a potential new therapeutic modality for epidemic of obesity diseases that are common and have only marginal therapies at this moment.

In summary, skeletal muscle-specific transgenic over-expression of PGC-1a in both transgenic mice and pigs results in significant oxidative/red fiber formation in major white muscle tissues. In compared with wild-type littermates, MHC type I fibers of gastrocnemius muscle was increased from 5\% to $50 \%$ of the total fibers in the transgenic mice. Results from gene expression analysis by $\mathrm{qPCR}$ and Western blotting supported increased expressions of oxidative fiber markers, and genes related glycogen deposition and fatty acid oxidation such as PDK4 and PPARY. Therefore, the results from both transgenic mice and pigs suggest that increased skeletal muscle-specific PGC-1aexpression promotes conversion of glycolytic fibers to oxidative fiber through enhanced mitochondrial respiration and fatty acid oxidation.

\section{Acknowledgements}

This work was supported by a grant from the National Project for Breeding of Transgenic Pig (2013ZX08006-002) to Huazhong Agriculture
University and an USDA Hatch Project (HAW00244-R) to the College of Tropical Agriculture and Human Resources of the University of Hawaii at Manoa.

\section{Competing Interests}

The authors have declared that no competing interest exists.

\section{References}

[1] Schiaffino S, Reggiani C. Myosin isoforms in mammalian skeletal muscle. J. Appl. Physiol. 1994;77:493-501

[2] Guth L, Samaha FJ. Qualitative differences between actomyosin ATPase of slow and fast mammalian muscle. Exp. Neurol. 1969; 25:138-152

[3] Schiaffino S, Reggiani C. Fiber Types in Mammalian Skeletal Muscles. Physiol. Rev. 2011;91:1447-1531

[4] Arany Z. PGC-1 coactivators and skeletal muscle adaptations in health and disease. Curr. Opin. Genet. Dev. 2008;18:426-434

[5] Channon AJ, Crompton RH, Günther MM, Vereecke EE. Muscle moment arms of the gibbon hind limb: implications for hylobatid locomotion. J. Anat. 2010;216:446-462

[6] Tseng YH, Cypess AM, Kahn CR. Cellular bioenergetics as a target for obesity therapy. Nat. Rev. Drug Discov. 2010;9: 465-482

[7] Arany Z, Lebrasseur N, Morris C, Smith E, Yang W, Ma Y, Chin S, Spiegelman $\mathrm{BM}$. The transcriptional coactivator PGC-1 $\beta$ drives the formation of oxidative type IIX fibers in skeletal muscle. Cell Metab. 2007;5: 35-46

[8] Grange RW, Meeson A, Chin E, Lau KS, Stull JT, Shelton JM, Williams RS,Garry DJ. Functional and molecular adaptations in skeletal muscle of myoglobin-mutant mice. Am. J. Physiol. - Cell Ph. 2001;281:1487-1494

[9] Booth FW, Baldwin KM. Muscle plasticity: energy demand and supply processes. Compr. Physiol. 2011;:1075-1123

[10] Pette D. The adaptive potential of skeletal muscle fibers. Can. J. Appl. Physiol. 2002; 27: 423-448

[11] Caiozzo VJ, Haddad F, Baker MJ, Herrick RE, Prietto N, Baldwin KM. Microgravity-induced transformations of myosin and contractile properties of skeletal muscle. J. Appl. Physiol. 1996;81:123-132

[12] Pette D, Staron RS. Mammalian skeletal muscle fiber type transitions. Int. Rev. Cytol. 1997; 170:143-223

[13] Puigserver P, Wu Z, Park CW, Graves R, Wright M, Spiegelman BM. A cold-inducible coactivator of nuclear receptors linked to adaptive thermogenesis. Cell. 1998;92:829-839

[14] Carmeliet P. Angiogenesis in health and disease. Nat. Med. 2003;9:653-660

[15] Herzig S, Long F, Jhala US, Hedrick S, Quinn R, Bauer A, Rudolph D, Schutz G, Yoon C, Puigserver P, Spiegelman B, Montminy M. CREB regulates hepatic gluconeogenesis through the coactivator PGC-1. Nature. 2001;413:179-183

[16] Puigserver P, Rhee J, Donovan J, Walkey CJ, Yoon JC, Oriente F, Kitamura Y, Altomonte J, Dong H, Accili D, Spiegelman BM. Insulin-regulated hepatic gluconeogenesis through FOXO1-PGC-1a interaction. Nature. 2003;423: 550-555

[17] Seale P, Kajimura S, Yang W, Chin S, Rohas LM, Uldry M, Tavernier G, Langin D, Spiegelman BM. Transcriptional Control of Brown Fat Determination by PRDM16. Cell Metab. 2007;6:38-54

[18] Kelly DP, Scarpulla RC. Transcriptional regulatory circuits controlling mitochondrial biogenesis and function. Gene. Dev. 2004;18:357-368

[19] Wu Z, Puigserver P, Andersson U, Zhang C, Adelmant G, Mootha V, Troy A, Cinti S, Lowell B,Scarpulla RC, Spiegelman BM. Mechanisms Controlling Mitochondrial Biogenesis and Respiration through the Thermogenic Coactivator PGC-1. Cell. 1999;98:115-124

[20] Lin J, Wu H, Tarr PT, Zhang CY, Wu Z, Boss O, Michael LF, Puigserver P, Isotani E, Olson EN, Lowell BB, Bassel-Duby R, Spiegelman BM. Transcriptional co-activator PGC-1 alpha drives the formation of slow-twitch muscle fibres. Nature. 2002;418:797-801

[21] Boquest AC, Grupen CG, Harrison SJ, McIlfatrick SM, Ashman RJ, d'Apice AJ, Nottle MB. Production of cloned pigs from cultured fetal fibroblast cells, Dynamic reprogramming of 5-hydroxymethylcytosine duringearly porcine embryogenesis. Theriogenology. 2014;81: 496-508

[22] Ogilvie RW, Feeback DL. Ametachromatic dye-ATPase method for the simultaneous identification of skeletal muscle fiber typesI, IIA, IIB and IIC. Biotech. Histochem. 1990;65:231-241

[23] Johnson JE, Wold BJ, Hauschka SD. Muscle Creatine Kinase Sequence Elements Regulating Skeletal and Cardiac Muscle Expression in Transgenic Mice. Mol. Cell. Biol. 1989; 9:3393-3399

[24] Morgunov I, Srere PA. Interaction between citrate synthase and malate dehydrogenase Substrate channeling of oxaloacetate. J. Biol. Chem. 1998;273: 29540-29544

[25] Kubis HP, Haller EA, Wetzel P, Gros G. Adult fast myosin pattern and ca2+ -induced slow myosin pattern in primary skeletal muscle culture. P. Natl. Acad. Sci. USA. 1997;94:4205-4210 
[26] Sonoda J, Mehl IR, Chong LW, Nofsinger RR, Evans RM. PGC-1beta controls mitochondrial metabolism to modulate circadian activity, adaptive thermogenesis, and hepatic steatosis. P. Natl. Acad. Sci. USA. 2007:104:5223-5228

[27] Wenz T, Diaz F, Spiegelman BM, Moraes CT. Activation of the PPAR/PGC-1a pathway prevents a bioenergetics deficit and effectively improves a mitochondrial myopathy phenotype. Cell Metab. 2008:8:249-256

[28] Austin S, Klimcakova E, St-Pierre J. Impact of PGC-1a on the topology and rate of superoxide production by the mitochondrial electron transport chain. Free Radical Bio. Med. 2011;51:2243-2248

[29] Soriano FX, Liesa M, Bach D, Chan DC, Palacin M, Zorzano A. Evidence for a mitochondrial regulatory pathway defined by peroxisome proliferator-activated receptor- $\gamma$ coactivator-1 1 , estrogen-related receptor- $a$, and mitofusin 2. Diabetes. 2006; 55:1783-1791

[30] Garnier A, Fortin D, Zolll J, N'Guessan B, Mettauer B, Lampert E, Veksler V, Ventura-Clapier R. Coordinated changes in mitochondrial function and biogenesis in healthy and diseased human skeletal muscle. FASEB J. 2005; $19: 43-52$

[31] Wende AR, Huss JM, Schaeffer PJ, Giguère V, Kelly DP. PGC-1a Coactivates PDK4 Gene Expression via the Orphan Nuclear Receptor ERRa: a Mechanism for Transcriptional Control of Muscle Glucose Metabolism. Mol. Cell. Biol. 2005;25:10684-10694

[32] Sugden MC, Holness MJ. Recent advances in mechanisms regulating glucose oxidation at the level of the pyruvate dehydrogenase complex by PDKs. Am. J. Physiol-Endoc. M. 2003;284:855-862

[33] Hoeks J, Arany Z, Phielix E, Moonen-Kornips E, Hesselink MKC, Schrauwen P. Enhanced lipid-but not carbohydrate-supported mitochondrial respiration inskeletal muscle of PGC-1a overexpressing mice. J. Cell. Physiol. 2012;227:1026-1033

[34] Yoon JC, Puigserver P, Chen G, Donovan J, Wu Z, Rhee J, Adelmant G, Stafford J, Kahn CR, Granner DK, Newgard CB, Spiegelman BM. Control of hepatic gluconeogenesis through the transcriptional coactivator PGC-1. Nature. 2001;413:131-138

[35] Randle PJ, Garland PB, Hales CN, Newsholme EA. The glucose fatty-acid cycle its role in insulin sensitivity and the metabolic disturbances of diabetes mellitus. Lancet.1963;281:785-789

[36] Evans RM, Barish GD, Wang YX. PPARs and the complex journey to obesity. Nat. Med. 2004;10:355-361

[37] Tontonoz P, Spiegelman BM. Fat and beyond: the diverse biology of PPARy. Annu. Rev. Biochem. 2008;77:289-312 